\title{
Schadet die Flexibilisierung des Arbeitsmarktes der Innovation?
}

Angelsächsische Länder mit flexiblen Arbeitsmärkten haben dasselbe Wirtschaftswachstum wie die korporatistischen Länder von „Old Europe“. Die Behauptung, dass „flexible“ Länder niedrigere Arbeitslosenzahlen hätten, ist zweifelhaft. Deregulierung von Arbeitsmärkten und Lohnzurückhaltung haben jedoch signifikant niedrigere Wachstumsraten der Arbeitsproduktivität zur Folge. Eine kürzere Verweildauer im Betrieb ist schädlich für Innovationen und Lernprozesse. Dieser Beitrag diskutiert theoretische Argumente, warum (von neoklassischen Ökonomen viel beklagte) „starre“ Arbeitsmärkte für Innovationen nützlich sein können. Empirische Studien fundieren diese Argumente.

ALFRED KLEINKNECHT, C. W. M. NAASTEPAD, SERVAAS STORM, ROBERT VERGEER

\section{Einleitung}

Seit den siebziger Jahren setzte sich die Sichtweise durch, dass Arbeitslosigkeit vor allem die Folge von unflexiblen, ja starren Arbeitsmärkten sei. Solche Starrheiten resultieren aus hohen Mindestlöhnen, großzügigen Sozialleistungen, der Macht von „Insidern“ oder einem starken Kündigungsschutz. Als eine weitere wichtige „Starrheit“ gelten die Gewerkschaften, die als Kartellorganisation die Löhne über dem Gleichgewichtsniveau halten und damit Menschen arbeitslos machen. Der Abbau solcher Starrheiten kann Löhne (nach unten) flexibel machen - und damit kann der Arbeitsmarkt als ein echter „Markt“ funktionieren, worin der Preis von Arbeit flexibel auf Schwankungen von Angebot und Nachfrage reagiert. Verbunden ist hiermit die Hoffnung auf eine niedrigere Arbeitslosigkeit.

Wie realistisch ist diese Perspektive? Dieser Artikel diskutiert theoretisch und empirisch begründete Zweifel an dieser Sichtweise. Unser Hauptargument lautet: Annahmen über die günstigen Effekte von „strukturellen Reformen“ zur Beseitigung von Arbeitsmarktrigiditäten wurden immer unter der (meist impliziten) Annahme gemacht, dass dies keinen Einfluss auf Innovationen habe. Wir argumentieren, dass dies nicht stimmt: Flexibilisierung von Arbeit zulasten der Arbeitnehmer ist vor allem schädlich für das Funktionieren des „routinemäßigen“ („Schumpeter II“) Innovationsmodells.
Der nächste Abschnitt dokumentiert einige makroökonomische Kernvariablen für die Periode 1960-2011 für eine Gruppe von typisch „rheinländischen“ Ländern des alten Europas (mit „rigiden“ Arbeitsmärkten) im Vergleich zu einer Gruppe typisch angelsächsischer Länder mit „liberalisierten" Arbeitsmärkten. Danach fassen wir theoretische Argumente zusammen, warum flexible Arbeit die Innovation negativ beeinflusst, und fundieren sie mit empirischen Befunden.

\section{Varieties of Capitalism: ein Ländervergleich}

Hall und Soskice (2001) unterscheiden zwei Typen von Arbeitsmarkt-Systemen: (1) Liberalized Market Economies (LME) versus (2) Coordinated Market Economies (CME). Die LME-Länder zeichnen sich durch einen lockeren Kündigungsschutz, bescheidene Sozialleistungen, schwache Gewerkschaften und dezentralisierte Tarifverhandlungen aus, die Ungleichheit in der Einkommensverteilung begünstigen. Gegenüber dem bescheidenen Schutz der Arbeit steht ein starker Schutz der Investoren. Typische LME-Länder sind die USA, Kanada, Großbritannien, Irland, Australien und Neuseeland. Für CME-Länder gilt das Gegenteil: starker Kündigungsschutz, großzügige Sozialleistungen, starke 
ABB. 1

\section{Entwicklung der Reallöhne 1960-2011}

$1960=100 \quad$ - Kontinentaleuropäische Länder —Angelsächsische Länder

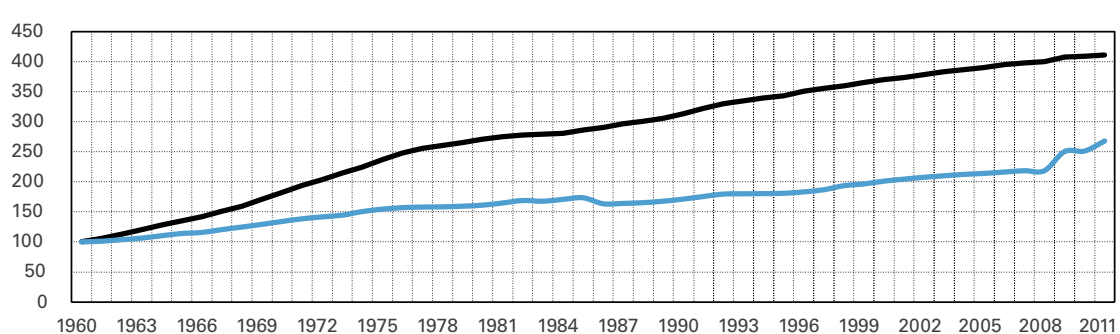

Kontinentaleuropäische Länder: Österreich, Belgien, Dänemark, Finnland, Frankreich, Deutschland, Italien, Niederlande, Portugal, Spanien, Schweden. Angelsächsische Länder: Australien, Kanada, Neuseeland, Großbritannien, USA. Reallöhne $=$ Labour Productivity per hour (GGDC; EKS) * Wage Share at factor costs (Eurostat)

Quelle: Datenbank des Groningen Growth and Development Centre (www.ggdc.net), AMECO Datenbank.

\section{ABB. 2}

\section{Entwicklung der Arbeitsstunden 1960-2011}

$1960=100$

— Kontinentaleuropäische Länder —Angelsächsische Länder

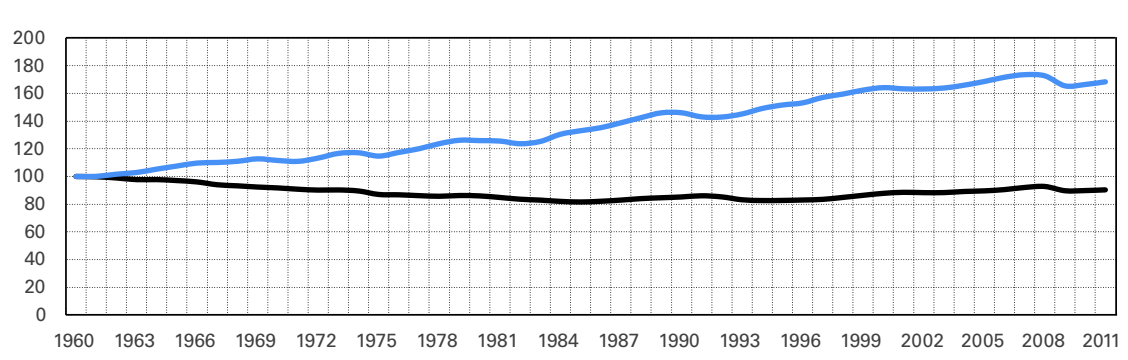

Kontinentaleuropäische Länder: Österreich, Belgien, Dänemark, Finnland, Frankreich, Deutschland, Italien, Niederlande, Portugal, Spanien, Schweden.

Angelsächsische Länder: Australien, Kanada, Neuseeland, Großbritannien, USA.

Quelle: Datenbank des Groningen Growth and Development Centre (www.ggdc.net).
Gewerkschaften sowie eine Tendenz zu zentralisierten Tarifverhandlungen und mehr Einkommensgleichheit. Gegenüber dem relativ starken Arbeitsschutz steht ein schwächerer Investorenschutz. Typische CME-Systeme findet man in Kontinentaleuropa und Japan (Hall/Soskice 2001).

In den folgenden Abbildungen werden vier wirtschaftliche Kernvariablen für eine Gruppe des „rheinländischen“ Europas mit fünf angelsächsischen Ländern verglichen. Für eine bessere Vergleichbarkeit von Entwicklungen sind alle Daten auf $1960=100$ gesetzt.

Die Grafiken zeigen folgende Muster:

- Die Reallöhne im „Alten Europa“ stiegen erheblich schneller als in der Gruppe der angelsächsischen Länder (Abbildung 1). Dies ist keine Überraschung, da die „starren“ Arbeitsmärkte den Faktor Arbeit viel besser schützen und ihm mehr Verhandlungsmacht bieten.

- Wie erwartet wurden die mäßigen Lohnsteigungen in den angelsächsischen Ländern im Vergleich zum alten Europa mit mehr Arbeitseinsatz (in Arbeitsstunden) belohnt ( $A b$ bildung 2).

- Abbildung 3 zeigt, dass sich das Wachstum des Bruttoinlandsprodukts zwischen beiden Länder-Gruppen nur geringfügig unterscheidet.

- Abbildung 4 zeigt die logische Konsequenz aus Abbildung 2 und 3. Wenn sich das Wachstum des Bruttoinlandsprodukts (BIP) kaum unterscheidet und wenn zugleich der Arbeitseinsatz in den angelsächsischen Ländern erheblich schneller steigt, dann kann dies nur ein langsameres Wachstum der Wertschöpfung (BIP) pro Arbeitsstunde bedeuten, also ein geringeres Wachstum der Arbeitsproduktivität in den angelsächsischen Ländern. Positiv formuliert: Liberalisierte Arbeitsmärkte schaffen mehr Arbeit. Negativ formuliert: Die Angelsachsen müssen für dasselbe Wirtschaftswachstum mehr Stunden arbeiten.

\section{Ist die Arbeitslosigkeit in Ländern mit flexiblen Arbeitsmärkten niedriger?}

Das relativ arbeitsintensive Wirtschaftswachstum als Folge eines geringeren Wachstums der Arbeitsproduktivität in den angelsächsischen Ländern weckt die Hoffnung, dass die Arbeitslosigkeit niedriger ausfallen könnte. Diese Hoffnung ist auch mit der Deregulierung der Arbeitsmärkte in Europa verbunden. Haben angelsächsische Länder in der Tat niedrigere Arbeitslosenquoten? Ein häufig zitierter Beitrag von Nickell et al. (2005) behauptet dies: Rigiditäten im Arbeitsmarkt erhöhen systematisch die Arbeitslosigkeit. Dieser Befund wurde wiederholt gebraucht, um die Forderung nach „strukturellen Reformen“ zur Deregulierung von Arbeitsmärkten zu stützen. Die Ergebnisse von Nickell et al. implizieren übrigens auch, dass die Gewerkschaften mit 
ihrer Opposition gegen die Deregulierung des Arbeitsmarktes eigentlich asozial seien: Sie verteidigen die Privilegien der „Insider" mit gesicherten Stellen und errichten Barrieren, die „Outsider" nicht überwinden könnten.

Diverse Studien ziehen dies in Zweifel und argumentieren, dass Schätzungen der Labour-Market-Rigidities-Anhänger nicht robust sind: Verändert man etwa den Beobachtungszeitraum oder die Anzahl der beobachteten Länder, dann ändern sich die Ergebnisse (Baker et al. 2005; Baccaro/ Rei 2007; Howell et al. 2007). Vergeer und Kleinknecht (2012a) haben die Modellschätzung von Nickell et al. (2005) einer Serie von Robustheitstests unterzogen. Die Ergebnisse sind überraschend. Während Nickell et al. (2005) finden, dass „rigide“ Arbeitsmarktinstitutionen systematisch (positiv) mit höheren Arbeitslosenquoten zusammenhängen, zeigen Vergeer und Kleinknecht (2012a), dass (mit exakt denselben Daten) auch ganz andere Ergebnisse herauskommen können. Eine Reihe von Koeffizienten im Modell von Nickell et al. (2005) wird insignifikant (und einige verändern sogar ihr Vorzeichen), wenn man nur drei kleine Details im Modell verändert - und für jedes dieser drei Details gibt es plausible Argumente. Mit anderen Worten, das viel zitierte Modell von Nickell et al. (2005) ist nicht robust. Es gibt also gute Gründe, das Postulat der Angebotstheoretiker anzuzweifeln, dass Unterschiede in der Höhe der Arbeitslosigkeit systematisch mit Indikatoren der Arbeitsmarktstarrheit zusammenhängen.

Tabelle 1 gibt langfristige Arbeitslosenzahlen in einigen LME- und CME-Ländern. Diese Zahlen illustrieren, dass es langfristig (1970 - 2010) keinesfalls eindeutig ist, welches der beiden Regime für Arbeitslose besser ist.

Wie kann man erklären, dass die angelsächsischen Länder trotz ihres arbeitsintensiven Wirtschaftswachstums (vgl. Abbildung 2) keine niedrigeren Arbeitslosenzahlen haben? Mögliche Erklärungen liegen in einer Vergrößerung des Arbeitsangebots durch eine großzügige Einwanderungspolitik und in längeren Arbeitszeiten (vgl. Tabelle 2). Für Arbeitgeber kann es vorteilhafter sein, das Personal länger arbeiten zu lassen, anstatt neues einzustellen. Die flexiblen Arbeitsmarktinstitutionen in den angelsächsischen Ländern geben den Arbeitgebern mehr Macht, längere Arbeitszeiten durchzusetzen.

Eine andere (komplementäre) Erklärung liegt in der Aufgabe der Zentralbank zur Bekämpfung der Inflation. Diverse Zentralbanken glauben inzwischen an die Theorie der NAIRU (=Non-Accelerating Inflation Rate of Unemployment). Anders formuliert: Es ist gewünscht, dass ein Land ein gewisses Niveau an Arbeitslosigkeit hat, sodass genügend Konkurrenz um knappe Stellen besteht. Nur so kann

1 Sturn (2011) argumentiert ähnlich. Er betont allerdings, dass der Einfluss von Arbeitsmarktrigiditäten auf die Arbeitslosigkeit auch abhängig ist vom dominanten Arbeitsmarktregime, wobei „institutionelle Komplementaritäten" zu berücksichtigen sind sowie die Rolle der Zentralbank.
ABB. 4

\section{Entwicklung der Arbeitsproduktivität (BIP pro Stunde) 1960-2011}

$1960=100$

- Kontinentaleuropäische Länder -Angelsächsische Länder

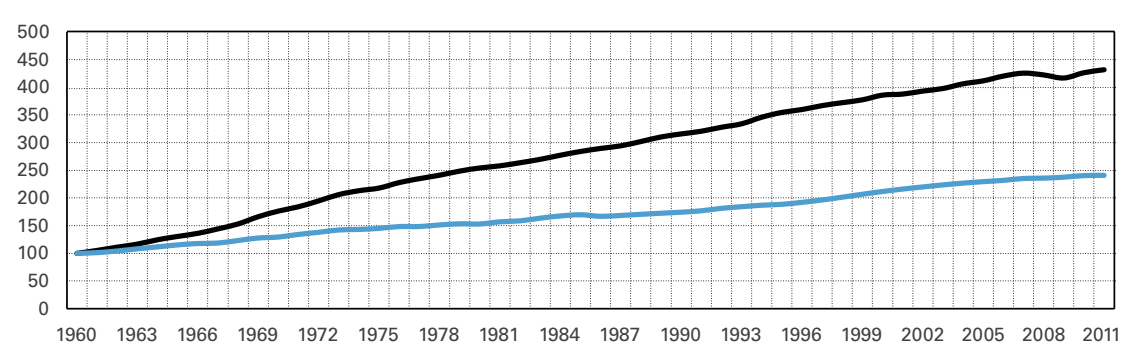

Kontinentaleuropäische Länder: Österreich, Belgien, Dänemark, Finnland, Frankreich, Deutschland, Italien, Niederlande, Portugal, Spanien, Schweden.

Angelsächsische Länder: Australien, Kanada, Neuseeland, Großbritannien, USA.

Quelle: Datenbank des Groningen Growth and Development Centre (www.ggdc.net).
TABELLE 1

\section{Durchschnittliche Arbeitslosigkeit 1970-2010}

Angaben in Prozent der aktiven Erwerbstätigen

\begin{tabular}{llll}
\hline \multicolumn{2}{c}{ „Flexible“ angelsächsische LME } & \multicolumn{2}{c}{ „Rigides Altes Europa“ (CME) } \\
\hline Kanada & 7,7 & Frankreich & 7,5 \\
\hline Großbritannien & 7,2 & Italien & 7,0 \\
\hline Australien & 6,3 & Deutschland & 6,2 \\
\hline USA & 6,3 & Niederlande & 6,1 \\
\hline & & Schweden & 4,7 \\
\hline Durchschnitt: & $\mathbf{6 , 9}$ & Durchschnitt: & $\mathbf{6 , 3}$ \\
\hline
\end{tabular}

Anmerkung: Alle Zahlen sind gemäß US Konvention definiert und damit vergleichbar.

Quelle: Bureau of Labor Statistics des US Department of Labor (2012)

http://www.bls.gov/fls/flscomparelf/tables.htm\#table01_ur.

\section{TABELLE 2}

\section{Arbeitsstunden pro Jahr 1990-2010}

Angaben in absoluten Zahlen

\begin{tabular}{llll}
\hline \multicolumn{2}{c}{ „Flexible“ angelsächsische LME } & \multicolumn{2}{c}{ „Rigides Altes Europa” (LME) } \\
\hline Australien & 1.767 & Italien & 1.840 \\
\hline Kanada & 1.761 & Schweden & 1.613 \\
\hline USA & 1.720 & Frankreich & 1.509 \\
\hline Großbritannien & 1.707 & Deutschland & 1.478 \\
\hline & & Niederlande & 1.432 \\
\hline Durchschnitt: & $\mathbf{1 . 7 3 9}$ & Durchschnitt: & $\mathbf{1 . 5 7 4}$ \\
\hline & & (Durchschnitt ohne Italien & $1.508)$ \\
\hline
\end{tabular}

Quelle:The Conference Board:Total economy database, Januar 2012: http://www.conference-board.org/data/economydatabase/. 
verhindert werden, dass Arbeitnehmer zu anspruchsvoll und Gewerkschaften zu mächtig werden, wodurch eine LohnPreis-Spirale entsteht. In den Niederlanden wird die „erwünschte" NAIRU-Arbeitslosenrate auf 4,5 \% geschätzt, in Deutschland etwas höher (Guichard/Rusicelli 2012). Sinkt die Arbeitslosenquote unter die NAIRU-Norm, dann sitzt die Wirtschaft in einer Gefahrenzone. Nach der Logik der NAIRU-Theorie muss dann die Zentralbank mit Zinserhöhungen eingreifen, um die „Überhitzung“ der Konjunktur und Inflation zu verhindern. Sollte es also gelingen, mit erfolgreicher Arbeitsmarktpolitik die Arbeitslosigkeit unter das NAIRU-Niveau zu senken, ist die Zentralbank gesetzlich verpflichtet einzugreifen. De facto bekämpft die Zentralbank also nicht die Inflation, sondern eine „zu niedrige“ Arbeitslosigkeit. Die gesetzliche Pflicht der Zentralbank, einzugreifen und zu verhindern, dass die Arbeitslosigkeit unter das NAIRU-Niveau fällt, ist eine mögliche Erklärung, warum Arbeitslosenzahlen in verschiedenen Ländern gar nicht so stark voneinander abweichen (vgl. Tabelle 1$){ }^{2}$

Während es zweifelhaft ist, ob die Arbeitslosenzahlen wirklich niedriger sind, haben die angelsächsischen Länder zweifellos eine stärkere Tendenz zu mehr Ungleichheit in der Einkommensverteilung. So ist der Anteil der $10 \%$ bestverdienenden Amerikaner am Nationaleinkommen von 33 \% im Jahre 1976 auf 50 \% im Jahre 2007 gestiegen. In der gleichen Periode stieg der Anteil der $1 \%$ reichsten Amerikaner von 8,9 auf 23,5 \% des Nationaleinkommens (Atkinson et al. 2011). In Europa hat die Ungleichheit erheblich weniger stark zugenommen. Der wirkliche Unterschied zwischen LMEs und CMEs liegt also nicht in den Arbeitslosenzahlen, sondern in den Einkommensunterschieden.

\section{Wie wirkt flexible Arbeit auf Innovation und Produktivität?}

Wir diskutieren nachfolgend Argumente für und gegen die Hypothese, dass flexible Arbeitsmärkte Innovation und Produktivität negativ beeinflussen. In diesem Zusammenhang sind für unsere Argumentation vor allem drei Arten von Flexibilität interessant: (1) Extern-numerische Flexibilität, die es Betrieben erlaubt, ihren Arbeitseinsatz durch flexible Einstellungen und Entlassungen anzupassen; (2) Lohnflexibilität: das Maß, in dem sich Löhne (nach unten) in Zeiten von Wirtschaftskrisen anpassen lassen; (3) funktionelle (oder interne) Flexibilität, die Betrieben die flexible Reallokation von Arbeit auf internen Arbeitsmärkten durch Weiterbildung und Personalpolitik erlaubt. Die beiden ersten Formen von Flexibilität sind charakteristisch für angelsächsische Länder, während die rheinländischen Modelle mehr auf funktionelle Flexibilität vertrauen (Hall/Soskice 2001).

Eine Reihe von Mikroanalysen zeigt, dass sich hohe Niveaus von funktioneller Flexibilität auf internen Arbeitsmärkten für Innovation und Arbeitsproduktivität günstig auswirken (Appelbaum et al. 2000; Bassanini/Ernst 2002; Michie/Sheehan 2001, 2003; Kleinknecht et al. 2006; Zhou et al. 2011). Die Anhänger der Arbeitsmarktderegulierung sind jedoch vor allem an Lohnkosten sparender numerischer Flexibilität und an (nach unten) flexiblen Löhnen interessiert.

Im Prinzip gehen „strukturelle Reformen“ mit dem Ziel flexibler Löhne und einer Lockerung des Kündigungsschutzes in dieselbe Richtung: Lohnmoderation oder gar -senkung. Wenn sich Arbeitsmärkte im Gleichgewicht befinden, würde man erwarten, dass Arbeitnehmer, die eine flexible Stelle akzeptieren, als Entschädigung für das höhere Arbeitsmarktrisiko einen normalen Lohn plus Risikoprämie erhalten. Empirische Lohnforschung zeigt jedoch eher das Gegenteil (Sànchez/Toharia 2000; Booth et al. 2002; McGinnity/Mertens 2004; Addison/Surfield 2005; Picchio 2006). Diesen Zusammenhang belegen auch Modellschätzungen auf Betriebsebene (Kleinknecht et al. 2006). Mit anderen Worten: Flexibel eingesetzte Arbeitnehmer verdienen durchschnittlich weniger als fest angestellte (nach Kontrolle für Alter, Branche, Geschlecht, Ausbildungsniveau, etc.). Eine mögliche Erklärung liegt im Arbeitsüberangebot in bestimmten Arbeitsmarktsegmenten (vor allem bei niedrig Qualifizierten). Für unsere Argumentation ist es unerheblich, ob ein geringeres Wachstum der Löhne durch institutionelle Reformen im System der Tarifverhandlungen, durch Lockerung des Kündigungsschutzes oder durch andere Reformen (etwa Senkung von Mindestlöhnen oder Sozialleistungen) erzielt wird.

Wir diskutieren nun drei Kanäle, durch die die Deregulierung von Arbeitsmärkten auf Innovation und Arbeitsproduktivität einwirkt:

- Auswirkungen auf die Innovationsaktivität,

- Auswirkungen auf Schulung,

- Auswirkungen auf Vertrauen und Loyalität.

\subsection{Auswirkungen auf die Innovation}

Drei Argumente weisen auf einen kausalen Einfluss von Löhnen auf die Arbeitsproduktivität hin. Zum einen kann eine Preiserhöhung für Arbeit (relativ zum Preis von Kapital) die Einführung von arbeitssparenden Innovationen fördern (Sylos Labini 1984, 1993, 1999). ${ }^{3}$ Zum Zweiten kann man argumentieren, dass dank ihrer Monopolgewinne aus der Innovation die Innovatoren besser als die technologischen Nachzügler mit Lohnerhöhungen leben können (oder

2 In der Studie von Guichard/Rusticelli (2012, S. 16-20) fällt auf, dass einige angelsächsische Länder (wie etwa Kanada) trotz ihrer flexiblen Arbeitsmärkte höhere Niveaus der NAI$\mathrm{RU}$-Arbeitslosigkeit haben als manche starren Länder (wie etwa Österreich). Auch dieser Befund sät Zweifel an der Behauptung, dass die NAIRU-Norm für Arbeitslosigkeit sinken kann, wenn die Arbeitsmärkte flexibler werden. Für eine umfassende Kritik an der NAIRU-Theorie siehe Storm/ Naastepad (2012). 
mit höheren Anpassungskosten durch striktere Regulierung). In diesem Sinne fördern Rigiditäten im Arbeitsmarkt Schumpeters Prozess der „schöpferischen Zerstörung“, worin die Innovatoren die Nachzügler wegkonkurrieren (Kleinknecht 1998). Oder umgekehrt: Dezentrale Lohnverhandlungen und (nach unten) flexible Löhne erhöhen die Wahrscheinlichkeit, dass die weniger talentierten Unternehmer überleben. Ihr Überleben ist möglicherweise kurzfristig günstig für die Beschäftigung; langfristig führt es jedoch zu einem Verlust an innovativer Dynamik.

Zum Dritten kann man mit Jahrgangsmodellen des Kapitalstocks leicht demonstrieren, dass eine aggressivere Lohnpolitik der Gewerkschaften ein schnelleres Ausrangieren von älteren (arbeitsintensiveren) Jahrgängen von Maschinen zugunsten neuer Jahrgänge mit höherer Produktivität zur Folge hat (Den Hartog/Tjan 1974; Muysken/van Ardenne 1976). Oder anders formuliert: Lohnzurückhaltung erlaubt es Betrieben, alte Jahrgänge länger profitabel zu benutzen. So ist das Resultat der Lohnzurückhaltung ein relativ älterer Kapitalstock - und dies war nach Naastepad/ Kleinknecht (2004) eine der Ursachen der niederländischen Produktivitätskrise in der langen Periode der Lohnzurückhaltung („Loonmatiging“) ab 1982.

Gegen diese Argumente gibt es in der Literatur drei Gegenargumente. Zum einen können „starre“ Arbeitsmärkte auf Innovation und Produktivität einen negativen Einfluss haben, da sie die Verschiebung von Arbeit aus alten, untergehenden Sektoren in neue, dynamische Sektoren behindern (Nickell/Layard 1999). Zum Zweiten kann schwierige und teure Entlassung von überflüssigem Personal negative Anreize für arbeitssparende Innovationen in Betrieben geben (Bassanini/Ernst 2002; Scarpetta/Tressel 2004). Zum Dritten können sich mächtige (weil gut geschützte) Arbeitnehmer und ihre Gewerkschaften mit ihren Lohnforderungen (Teile der) Monopol-Gewinne aus der Innovation aneignen. Damit verschwinden die Anreize, um innovative Risiken zu akzeptieren (Malcomson 1997).

Das letzte Argument ist zweifellos für die in angelsächsischen Ländern typischen dezentralen Tarifverhandlungen relevant. Es ist weniger relevant in den als rigide bezeichneten rheinländischen Systemen, wo mehr zentral (oft auf Branchenniveau) verhandelt wird. Das Argument, dass teure Entlassungen arbeitssparende Innovationen verzögern, kann aus drei Gründen weniger relevant sein: Erstens werden Betriebe, die nicht leicht entlassen können, dadurch motiviert, in funktionelle Flexibilität durch Weiterbildung zu investieren, wodurch Menschen leichter von alten in neue Tätigkeitsbereiche gelenkt werden können. Mit anderen Worten: Der Mangel an numerischer Flexibilität fördert funktionelle Flexibilität. ${ }^{4}$

Zweitens muss in vielen Ländern überzähliges Personal kein Problem für arbeitssparende Innovationen darstellen, da ein hoher Anteil der Beschäftigten freiwillig kündigt. ${ }^{5}$ Drittens kann man argumentieren, dass Kündigungsschutz die Produktivität auch erhöhen kann. Arbeitnehmer mit einer sicheren Stelle sind eher bereit, bei der Einführung von arbeitssparenden Technologien mit dem Management zusammenzuarbeiten. Für die erfolgreiche Einführung von Automatisierungstechnologie ist oft die langfristig aufgebaute persönliche Erfahrungskenntnis der Mitarbeiter nötig (Lorenz 1999). Müssen sie Entlassungen befürchten, dann kooperieren sie nicht. Überhaupt gilt, dass Mitarbeiter, die man leicht entlassen kann, ein Motiv haben, Informationen, wie z. B. ihre Arbeit effizienter gestaltet werden kann, zurückzuhalten. In einem flexiblen „hire \& fire“ System läuft man Gefahr, die Erfahrungskenntnisse der Mitarbeiter nur unzulänglich zu nutzen. Mitarbeiter machen dabei Gebrauch von Informations-Asymmetrien zwischen ihnen und dem Management.

\subsection{Auswirkungen auf Weiterbildung}

Ein lockerer Kündigungsschutz führt zu kürzeren Dienstverhältnissen, wodurch die Wahrscheinlichkeit abnimmt, dass sich Investitionen des Betriebes in Aus- und Weiterbildung bezahlt machen. Außerdem sind dann auch die Arbeitnehmer mehr an allgemeiner Weiterbildung interessiert, die die Beschäftigungschancen auf dem externen Arbeitsmarkt verbessert. Sie sind weniger an betriebsspezifischer Schulung interessiert, wenn keine Perspektive besteht, länger zu bleiben (Belot et al. 2002).

Ähnliche Argumente kann man aus der Hypothese ableiten, dass durch die Deregulierung von Arbeitsmärkten die Nivellierung („Compression“) der Lohnstruktur (innerhalb und zwischen Betrieben) vermindert wird - nach Acemoglu und Pischke (1999) und Agell (1999) ist diese Nivellierung ja gerade ein Grund, Weiterbildung anzubieten. Empirische Nachweise einer Korrelation zwischen Zeitverträgen und geringerer Wahrscheinlichkeit der Weiterbildung am Arbeitsplatz liefern für Großbritannien Arulampalam und Booth (1998) und Booth et al. (2002).

\subsection{Vertrauen und Innovation}

Arbeiten von Huselid (1995), Buchele/Christiansen (1999), Lorenz (1999), Michie/Sheehan $(2001,2003)$ und Naas-

\section{Man muss sich klar machen, dass sich die dynamische Substitution zwischen Kapital und Arbeit in diesem Kontext unterscheidet von der statischen Substitution (mit konstan- terTechnologie) in der neoklassischen Theorie als Reaktion auf eine Variation der relativen Faktorpreise. Die dynami- sche Substitution beruht auf technologischem Wandel, der in neuen Kapitalgütern inkorporiert ist (Sylos Labini 1993).}

4 Siehe auch Acemoglou/Pischke (1999), die argumentieren, dass die „wage compression" in den rigiden deutschen Arbeitsmärkten Training für höher und niedriger Ausgebildete fördert, während die liberalisierten Arbeitsmärkte in den USA vor allem Training für höher Ausgebildete begünstigen.

5 Kleinknecht et al. (2006) zeigen, das durchschnittlich 9-12\% des Personals in den Niederlanden jedes Jahr ihren Betrieb freiwillig verlassen, wobei die Quote vom Konjunkturzyklus abhängt. Nickell/Layard kommen auf 10 \% (1999, S. 363). 
tepad /Storm (2006) zeigen positive Produktivitätseffekte einer vertrauensvollen („high road“) Personalpolitik. Längerfristige Arbeitsbeziehungen und einen guten Kündigungsschutz kann man als eine Investition in Vertrauen (siehe auch Svensson 2011), Loyalität und Motivation interpretieren. Diese Faktoren haben in mehrerer Hinsicht günstige Effekte auf Innovation und Produktivität:

(1) Sie reduzieren Kosten für Aufsicht und Kontrolle. So zeigen Naastepad und Storm, dass Betriebe in angelsächsischen Ländern viel aufwändigere Management-Bürokratien unterhalten, um ihr Personal zu überwachen (2006, S. 170ff.).

(2) Sie reduzieren positive externe Effekte. Wenig loyale Mitarbeiter mit geringer Bindung an den Betrieb können Betriebsgeheimnisse und technologische Kenntnisse eher an Konkurrenten durchgeben und diese externen Effekte machen Investitionen in Kenntnisse weniger attraktiv.

(3) Sie begünstigen die langfristige Akkumulation von Kenntnissen - vor allem von wenig dokumentiertem und personengebundenem Erfahrungswissen („tacit knowledge"). Letzteres ist besonders in einem „Routinemodell der Innovation“ (Schumpeter 1943) wichtig. Die Eigenschaften des „Routinemodells“ im Vergleich zum „Unternehmermodell“ (Schumpeter 1912) sind abgebildet in Übersicht 1, die durch die Arbeit von Breschi et al. (2000) inspiriert ist.

(4) Bei gutem Kündigungsschutz und Vertrauen bekommen Vorgesetzte mehr kritisches Feedback von unten. Mächtige Manager neigen zuweilen dazu, sich mit Konformisten zu umgeben. Sie entwickeln dadurch auf Dauer ein verzeichnetes Bild der Realität. Wird non-konformistisches Verhalten noch durch die Drohung mit leichter Entlassung verstärkt, dann kann das problematisches Managementverhalten an der Spitze der Hierarchie verstärken („Sonnenkönige“).
Aus Übersicht 1 wird deutlich, dass Schumpeters Routinemodell besser mit langfristigen und vertrauensvollen Arbeitsbeziehungen funktioniert. Das Routinemodell gibt Anreize für die Re-Allokation von Arbeit in internen Arbeitsmärkten durch funktionelle Flexibilität anstelle von numerischer Flexibilität durch Entlassung und Einstellung auf externen Arbeitsmärkten. Vor allem der kumulative Charakter der Kenntnisse schafft starke Pfadabhängigkeiten in der betrieblichen Wissensbasis und macht „Insider“Arbeitsbeziehungen attraktiv für Arbeitgeber.

\section{Empirische Beobachtungen}

Die meisten empirischen Studien zur Beziehung zwischen flexibler Arbeit und dem Wachstum der Arbeitsproduktivität verwenden Länder- oder Sektordaten (Buchele/Christiansen 1999; Nickell/Layard 1999; Bassanini/Ernst 2002; Scarpetta/Tressel 2004; Auer et al. 2005; Naastepad/Storm 2006). Meistens sucht man nach einer Beziehung zwischen Maßstäben der Arbeitsmarktrigidität (wie etwa dem EPL: Employment Protection Legislation Index der OECD) einerseits und andererseits dem Produktivitätswachstum. Nicht alle, aber die meisten Studien finden einen positiven Einfluss der Arbeitsmarktrigidität auf das Wachstum der Arbeitsproduktivität (für das Wachstum der totalen Faktorproduktivität sind die Ergebnisse nicht eindeutig, siehe Vergeer 2010). Die Studie von Bassanini et al. (2009) hingegen suggeriert, dass der Netto-Effekt der Arbeitsmarktregulierung auf das Wachstum der Arbeitsproduktivität negativ ist. Eine frühere Version der Studie von Bassanini et al. (2009) lieferte Input für den OECD Employment Outlook (2007). In Letzterem steht, dass der Netto-Effekt der Arbeitsmarktregulierung auf das Produktivitätswachstum signifikant negativ (aber klein) ist.

ÜBERSICHT 1

\section{Stilisierter Vergleich der zwei Innovationsmodelle von Schumpeter (1912 und 1943)}

\begin{tabular}{|c|c|}
\hline $\begin{array}{l}\text { Schumpeter I Modell (1912): } \\
\text { "Garage Business Innovation" }\end{array}$ & $\begin{array}{l}\text { Schumpeter II Modell (1943): } \\
\text { „Routinemodell der Innovation“ }\end{array}$ \\
\hline Starter in high tech; Nischenspieler & Etablierte Betriebe mit professionellen R\&D Laboratorien \\
\hline KMU und junge Betriebe & Monopolistische Konkurrenz, Oligopole \\
\hline Hohe Zu- und Austrittsraten & Stabile Hierarchie von (dominanten) Innovatoren \\
\hline \multicolumn{2}{|c|}{ Eigenschaften der Kenntnisbasis ... } \\
\hline $\begin{array}{l}\text { Allgemeine und spontan mobilisierbare Kenntnisse } \rightarrow \text { niedri- } \\
\text { ge Barrieren für Markteintritt }\end{array}$ & 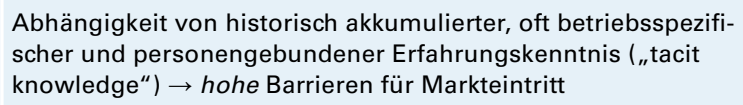 \\
\hline \multicolumn{2}{|c|}{... und dazu passende Arbeitsmarktinstitutionen } \\
\hline Anwerbung über externe Arbeitsmärkte & Interne Arbeitsmärkte $\rightarrow$ gut geschützte „Insider" \\
\hline
\end{tabular}


Zwei andere OECD-Forscher, Scarpetta und Tressel (2004), finden einen starken negativen Einfluss von Arbeitsmarktrigiditäten auf das Produktivitätswachstum in einem Teil der OECD-Länder (d.h. in Ländern mit Tarifverhandlungen auf Industrieniveau, jedoch ohne nationale Koordination); für alle OECD-Länder zusammen zeigt sich jedoch kein Einfluss. Diese Schlussfolgerung ist jedoch nicht sehr verlässlich, da der empirische Forschungsansatz wichtige Mängel hat. ${ }^{6}$

Dahingegen finden Auer et al. (2005) eine positive (obwohl abnehmende) Beziehung zwischen stabilen Beschäftigungsverhältnissen (gemessen an der Dauer der Beschäftigung) und der Arbeitsproduktivität. Vergeer und Kleinknecht (2012b) gebrauchen in einer Panelanalyse von 20 OECD-Ländern von 1960 - 2004 nicht den EPL-Maßstab (für den Kündigungsschutz), sondern zwei andere Maßstäbe: prozentuale Lohnerhöhungen und den Anteil der Kapitaleinkommen am Volkseinkommen. Nach Korrektur einer Reihe von anderen einflussreichen Faktoren (wie etwa: Anteil der Dienstleister in der gesamten Wirtschaft; Verdoorn-Effekt; Aufholeffekte von niedriger entwickelten Ländern, usw.) kommen sie zum Ergebnis:

- $1 \%$ extra Lohnerhöhung (bzw. Lohnsenkung) führt zu $0,35-0,45 \%$ zusätzlichem (bzw. weniger) Wachstum der Arbeitsproduktivität.

- $1 \%$ Steigerung des Anteils der Kapitaleinkommen am Volkseinkommen bedeutet ca. 0,2 \% weniger Wachstum der Arbeitsproduktivität. Da im OECD-Durchschnitt ab Mitte der siebziger Jahre der Anteil des Kapitaleinkommens am Volkseinkommen ca. 7 \% gestiegen ist, erklärt dies eine Reduktion des jährlichen Wachstums der Arbeitsproduktivität um ca. $1,4 \%$.

Eine andere Untersuchung von Acharya et al. (2010) nutzt Daten über Patente und Patentzitate (Letztere als Maßstab für die Qualität von Patenten). Die Autoren argumentieren, dass guter Kündigungsschutz Sicherheit gibt, dass
Mitarbeiter für misslungene Projekte nicht mit Entlassung bestraft werden. Dadurch getrauen sich Angestellte bei der Suche nach innovativen Lösungen eher riskante (aber potenziell sehr lohnende) Projekte in Angriff zu nehmen. Aus der Analyse von Zeitreihenvariationen (entstanden durch Veränderungen im Arbeitsrecht) ziehen sie die Schlussfolgerung, dass „[...] innovation and growth are fostered by stringent laws governing dismissal of employees, especially in the more innovation-intensive sectors. Firm-level tests within the United States that exploit a discontinuity generated by the passage of the federal Worker Adjustment and Retraining Notification Act confirm the cross-country evidence." (2010, S. 1).

Auch Pieroni and Pompei (2008) gebrauchen Patentdaten. Sie finden im Vergleich zwischen italienischen Regionen, dass häufiger Arbeitsplatzwechsel zwischen Betrieben (als Maßstab für numerische Flexibilität) einen negativen Effekt auf Patentaktivitäten hat.

Es gibt auch Studien auf Unternehmensebene. Zum Beispiel finden Michie und Sheehan $(2001,2003)$ in britischen Betrieben eine positive Korrelation zwischen „high road“ Personalpolitik und Innovation. Kleinknecht et al. (2006) zeigen für niederländische Industriebetriebe negative Effekte bei externer Flexibilität (Zeitverträge) und positive Effekte bei funktioneller Flexibilität (Mobilität auf internen Arbeitsmärkten) auf das Wachstum der Arbeitsproduktivität. Arvanitis (2005) kommt für schweizerische Betriebe ebenfalls auf eine positive Beziehung zwischen funktioneller Flexibilität und Arbeitsproduktivität. Für externe Flexibilität ist der Effekt jedoch insignifikant.

Autor et al. (2007) finden einen positiven Effekt von Kündigungsschutz auf Investitionen in Kapitalgüter, Qualifikation und Arbeitsproduktivität sowie einen negativen Effekt auf die totale Faktorproduktivität.

Lucidi und Kleinknecht (2010) kommen in ihrer Untersuchung auf Basis von 3.000 italienischen Industriebetrieben zu dem Ergebnis, dass ein hoher Anteil von flexiblen Arbeitskräften (im Jahre 2001), ein großer Wechsel
6 Die grundlegende (aber nicht getestete) Idee von Bassanini et al. (2009) ist, dass der Kündigungsschutz (gemessen an dem EPL-Index) Industrien mit hohen Entlassungsraten stärker beeinflusst als solche mit niedrigen Entlassungsraten. Sie gebrauchen Daten von 17 Industrien aus 18 OECD-Ländern (1982-2003) und klassifizieren Industrien in "bindende" und "nicht bindende" Industrien, wobei die Letzteren als Kontrollgruppe dienen. Zum Ersten ist die Klassifikation dieser beiden Gruppen keineswegs eindeutig. Es unterstellt immerhin, dass es (ohne Kündigungsschutz) so etwas wie eine "natürliche" Entlassungsrate gibt. Zum Zweiten ist es unrealistisch zu unterstellen, dass die Klassifikation in „bindende" und "nicht bindende" Industrien (i) für alle Jahre zwischen 1982 und 2003 gilt, und (ii), dass es keine Unterschiede zwischen den Ländern gibt; von allen Ländern wird angenommen, dass sie den USA ähnlich sind. Die USA dienen als Benchmark, womit länderspezifische institutionelle Faktoren, die die „natürlichen“ Entlassungsraten beeinflussen, negiert werden. Ein drittes Problem ist, dass die länder- spezifischen EPL-Indizes sich im Laufe der Zeit wenig ändern. Das bedeutet, dass ein Großteil der Unterschiede in den EPL aus Unterschieden zwischen Ländern kommt. Der EPL-Unterschied innerhalb der Länder kann also nicht viel über die länderspezifischen Produktivitätsdifferenzen in den verschiedenen Industrien erklären. Zum Schluss liegt der Nachdruck der Analyse auf Unterschieden in Produktivitätswachstumsraten zwischen "bindenden" und "nicht bindenden“ Industrien. Um dann den Einfluss der EPL auf das gesamtwirtschaftliche Wachstum der Arbeitsproduktivität zu bestimmen, werden potenzielle allgemeine GleichgewichtsEffekte der EPL "wegunterstellt" (wie etwa: der Einfluss der EPL auf (a) das Produktivitätswachstum in "nicht-bindenden“ Industrien, (b) das relative Gewicht (in Wertschöpfung) von „bindenden" und "nicht-bindenden" Industrien und (c) Anreize für Investitionen in physisches und menschliches Kapital. Alles in allem ist das Resultat, dass der Kündigungsschutz das Wachstum der Arbeitsproduktivität behindert, nicht sehr überzeugend. 
von Arbeitskräften (im Jahre 2001) sowie relativ niedrige Arbeitskosten (relativ zu Kapitalkosten, 1998 - 2000) jeweils die Wachstumsraten der Arbeitsproduktivität 2001-2003 negativ beeinflussen. Boeri und Garibaldi (2007) finden einen negativen Effekt des Anteils von Zeitverträgen auf das Wachstum der Arbeitsproduktivität in italienischen Industriebetrieben im Zeitraum von 1995-2000.

Viele der oben genannten Argumente unterstellen (implizit), dass ein geringer Kündigungsschutz zu häufigerem Arbeitsplatzwechsel führt. Hiergegen könnte man einwenden, dass eine gesetzlich geregelte Lockerung des Kündigungsschutzes die Betriebe nicht hindern muss, ihr Personal länger zu beschäftigen. Dagegen ließe sich allerdings sagen: Allein schon die gesetzliche Option einer leicht möglichen Entlassung macht Menschen mobiler. Geht es zum Beispiel mit einem Betrieb nicht so gut, dann kann es wichtig sein, so früh wie möglich auf Jobsuche zu gehen. Sobald nämlich tatsächlich Massenentlassungen stattfinden, nimmt im regionalen Arbeitsmarkt die Konkurrenz um offene Stellen dramatisch zu. So kann es geschehen, dass vorausschauende Menschen den Betrieb verlassen, obwohl dieser sie gar nicht entlassen wollte.

\section{Abschließende Diskussion}

Die obigen Ausführungen erlauben eine Reihe von Schlussfolgerungen über die Effekte von „strukturellen Reformen“, die Rigiditäten am Arbeitsmarkt beseitigen:

Erstens, langfristig unterscheidet sich das Wirtschaftswachstum in „liberalisierten“ Marktwirtschaften (LME) nur geringfügig vom Wachstum der „koordinierten“ Marktwirtschaften (CME). Zuweilen wurde behauptet, dass die Deregulierung von Faktormärkten (und insbesondere von $\mathrm{Ar}$ beitsmärkten) das relativ hohe Wirtschaftswachstum in den USA in den neunziger Jahren (bis 2007) erkläre. Vergleicht man jedoch eine Gruppe von LME (Australien, Kanada, NeuSeeland, Großbritannien und USA) mit einer Gruppe von CME (Österreich, Belgien, Dänemark, Finnland, Frankreich, Deutschland, Italien, Niederlande, Portugal, Spanien, und Schweden), sind kaum nennenswerte Unterschiede zu sehen (Abbildung 3). Inzwischen wissen wir, dass das hohe Wachstum in den USA vor 2007 viel mit einem eindrucksvollen Schuldenaufbau zu tun hatte (Maki/Palumbo 2001; Palley 2009; Irvin 2011). Der Schuldenaufbau ging mit Seifenblasen auf dem Häusermarkt einher, wodurch Amerikaner ihr Haus als Geldautomat gebrauchen konnten. Man könnte dies auch „Hypotheken-Keynesianismus“ nennen.

Zweitens haben die angelsächsischen LME im Vergleich zum alten Europa ein relativ bescheidenes Reallohn-Wachstum. Offensichtlich bieten liberalisierte Arbeitsmärkte (mit schwachen Gewerkschaften) einen schwachen Schutz für Arbeit. Dies hat - verglichen mit dem alten Europa - eine stark wachsende Einkommensungleichheit zum Ergebnis.
Drittens, wie man aus der traditionellen mikroökonomischen Theorie erwarten konnte, führt ein schwaches Lohnwachstum zu einem arbeitsintensiven Wirtschaftswachstum, und dies geht auf Kosten eines geringeren Wachstums der Wertschöpfung pro Arbeitsstunde. Die Ironie der Geschichte will, dass uns dies an das faktorintensive Wachstum in Osteuropa vor 1989 erinnert. Mit Blick auf die relative Überalterung der Bevölkerung und eine schrumpfende aktive Berufsbevölkerung stellt sich die Frage, ob Arbeitsbeschaffungsmaßnahmen im alten osteuropäischen Stil in Westeuropa gewünscht sind.

Viertens, obwohl die Arbeitslosenzahlen in den USA (jedenfalls in der Periode des „Hypotheken-Keynesianismus“ vor 2007) relativ gut aussahen, stellt sich langfristig die Frage, ob die Zahlen für die Gruppe von LME auch wirklich besser aussehen als in den CME (Tabelle 1). Mehrere Autoren kommen zu der Schlussfolgerung, dass es zweifelhaft ist, ob Deregulierung von Arbeitsmärkten zu niedrigerer Arbeitslosigkeit führt (und das trotz des relativ arbeitsintensiven Wachstums; siehe Baker et al. 2005; Baccaro/Rei 2007; Howell et al. 2007). Selbst bei der viel zitierten (und politisch sehr einflussreichen) Studie von Nickell et al. (2005) kann man zeigen, dass sie auf nicht-robusten Schätzungen beruht (Vergeer/Kleinknecht 2012a).

Die Fairness gebietet es hinzuzufügen, dass viele Forscher in der "Arbeitslosigkeit-durch-starre-Arbeitsmärkte“Literatur ihr Bestes getan haben zu betonen, dass es bei ihrer Arbeit allerlei Probleme und Beschränkungen gibt. Vielfach wurde auf Unzulänglichkeiten der verfügbaren $\mathrm{Da}$ ten und Grenzen der ökonometrischen Methoden verwiesen. Dies hat jedoch resolute Politikempfehlungen durch politiknahe Funktionäre nicht verhindert!

Fünftens haben wir Argumente vorgetragen, warum deregulierte Arbeitsmärkte für Innovationen und Lernprozesse schädlich sind. Diese Argumente sind vor allem für ein „routinemäßiges“ Innovationsmodell relevant (Schumpeter 1943). Dieses Modell beruht auf einer relativ pfadabhängigen Akkumulation von (oft betriebsspezifischem) und schlecht dokumentiertem (personengebundenem) Erfahrungswissen („tacit knowledge“, siehe Polanyi 1966). Hier bieten Arbeitsmärkte mit relativ geringer Mobilität Vorteile. Das kann eine Erklärung sein, warum große Teile der amerikanischen Industrie so schlecht gegen deutsche und japanische Anbieter konkurrieren können. Die USA sind relativ stark in der Informationstechnologie. Hier gilt das „unternehmerische“ („Garage-business") Modell der Innovation (Schumpeter 1912). Dieses Modell gebraucht spontan mobilisierbare, allgemeine Kenntnisse und hat weniger Probleme mit flüchtigen und flexiblen Arbeitsverhältnissen. Allerdings ist nicht auszuschließen, dass die erfolgreichen Betriebe in der amerikanischen IT-Industrie im Laufe ihrer Entwicklung auch Züge des routinemäßigen SchumpeterII-Modells (Schumpeter 1943) entwickeln - und dann kann der hire and fire-Arbeitsmarkt nachteilig wirken.

Zum Schluss noch ein Wort zu einer Hypothese, die unseren obenstehenden Argumenten zu widersprechen 
scheint: Kann das langsamere Wachstum der Arbeitsproduktivität nicht auch aus dem Umstand erklärt werden, dass die Deregulierung von Arbeitsmärkten einen kräftigen Zustrom von niedrig qualifizierten Arbeitskräften auf den Arbeitsmarkt ermöglicht? Die OECD (2003) formuliert diese growth-in-low-productive-jobs-Hypothese wie folgt: Es kann durchaus einen (leichten) „Trade-off“ geben zwischen dem zusätzlichen Wachstum der Beschäftigung und dem der Produktivität. Ausschlaggebend sind neue Jobs im Niedriglohn-Segment des Arbeitsmarktes: „For example, decentralization of wage bargaining and trimming back of high minimum wages may tend to lower wages, at least in the lower ranges of the earnings distribution. Similarly, relaxing employment protection legislation [...] may encourage expansion of low-productivity/low-pay jobs in services." (OECD, 2003; Box 1.4, S. 42.).

Diese niedrigproduktiven Jobs, wird dann behauptet, würden in flexiblen Ländern geschaffen. In rigiden Ländern verbannen ja die hohen Mindestlöhne und Sozialleistungen die Niedrigproduktiven vom Arbeitsmarkt. Aus dieser Sicht ist dann das geringere Wachstum der Arbeitsproduktivität durch Deregulierung lediglich ein Nebenprodukt von Extrajobs im Segment der Niedriglöhne.

Zwei Gegenargumente: Erstens, unter der ceteris-paribus-Annahme kann man argumentieren, dass die Mobilisierung von Extra-Arbeitskräften in den flexiblen Ländern zu Extra-Wirtschaftswachstum führen müsste im Vergleich zu den rigiden Ländern, wo diese Leute nicht arbeiten. Hierfür gibt es jedoch kaum Belege (vgl. Abbildung 3). Zweitens, die low productive-jobs-Hypothese wurde im obengenannten Modell von Vergeer und Kleinknecht (2012b) getestet. In der Analyse von Faktoren, die das Wachstum der Arbeitsproduktivität in 20 OECD-Ländern (1960 - 2004) erklären, ist u. a. das Wachstum des Arbeitseinsatzes als erklärende Variable aufgenommen. Mit anderen Worten: Es wurde getestet, ob ExtraJobwachstum (durch Zulassung von niedrigproduktiven Arbeitskräften im Arbeitsmarkt) das Wachstum der Arbeitsproduktivität negativ beeinflusst. Wie erwartet hatte der Koeffizient der Wachstumsrate des Arbeitseinsatzes ein negatives Vorzeichen. Der Effekt ist allerdings weit entfernt von jeglicher statistischer Signifikanz. Dies stützt die Vermutung, dass das schwache Wachstum der Arbeitsproduktivität in deregulierten Arbeitsmärkten kaum durch die low-productive-jobs-Hypothese der OECD zu erklären ist. Die Erklärung muss vor allem in Faktoren gesucht werden, die das geringere Wachstum der Arbeitsproduktivität bei schon bestehenden Arbeitsplätzen erklären.

Relevante Erklärungen für das Letztere sind die geringere Substitution von Arbeit durch Kapital, Jahrgangs-Effekte, die an Überalterung des Kapitalstocks leiden, oder weniger „schöpferische Zerstörung“ im Sinne von Schumpeter, da Deregulierung von Arbeitsmärkten die Überlebenschancen von schwächeren Unternehmern erhöht. Außerdem sorgt die Flexibilisierung für kürzere, flüchtigere Arbeitsbeziehungen, wodurch betriebliche Investitionen in Fortbildung weniger lohnen und die Basis für Vertrauen und Loyalität abnimmt. Dies zwingt Betriebe in aufwendigere ManagementBürokratien zu investieren. Ein großes Problem ist dabei, dass das „routinemäßige“ Innovationsmodell nicht mehr gut funktioniert. Für die pfadabhängige Akkumulation von personengebundener Betriebserfahrung hat dieses Modell personelle Kontinuität nötig.

Zusammenfassend kann mit Milton Friedman wieder einmal konstatiert werden: „There is no such thing as a free lunch.“ Die Deregulierung von Arbeitsmärkten hat ihren Preis. Aus der neoklassischen Mikroökonomie kann man mit großer Überzeugungskraft ableiten, dass Arbeitsmärkte eigentlich nie flexibel genug sein können. Die neoklassische Theorie hat jedoch keine Theorie der (routinemäßigen) Innovation. Was aus neoklassischer Sicht vernünftig ist, kann aus schumpeterianischer Innovationsperspektive kontraproduktiv sein.

\section{LITERATUR}

Acemoglou, D./Pischke, J.-S. (1999): Beyond Becker:Training in Imperfect Labour Markets, in: Economic Journal 109 (453), S. 112-142

Acharya, V. V./Baghai, R. P./Subramanian, K. V. (2010): Labor laws and innovation: National Bureau of Economic Research, NBER Working Paper (16484),

Cambridge, MA

Addison, J. T./Surfield, C. J. (2005): Atypical Work and Compensation, IZA Discussion Paper (1477)

Agell, J. (1999): On the benefits from rigid labour markets: Norms, market failures, and social insurance, in: Economic Journal 109 (453), S. 143-164

Appelbaum, E./Bailey, T./Berg, P./Kalleberg, A. L. (2000): Manufacturing advantage. Why high-performance work systems pay off, Ithaca, NY

Arulampalam ,W./Booth, A. L. (1998), Training and Labour Market Flexibility: Is There aTrade-off?, in: British Journal of Industrial Relations 36 (4), S. 521-536 Arvanitis, S. (2005): Modes of labor flexibility at firm level: are there any implications for performance and innovation? Evidence for the Swiss economy, in: Industrial and Corporate Change 14 (6), S. 993-1016

Atkinson, A./Piketty, T./Saez, E. (2011): Top incomes in the long run of history, in: Journal of Economic Literature 49 (1), S. 3-71

Auer, P./Berg, J./Coulibaly, I. (2005): Is a stable workforce good for productivity?, in: International Labour Review 144 (3), S. 319-343

Autor, D. H./Kerr W. R./Kugler, A. D. (2007): Does Employment Protection Reduce Productivity? Evidence from US States, in: Economic Journal 117 (521), S. $189-217$

Baccaro, L./Rei, D. (2007): Institutional determinants of unemployment in OECD countries: Does the deregulatory view hold water? in: International Organization 61 (3), S. 527-569

Baker, D./Glyn, A./Howell, D./Schmitt, J. (2005): Labor market institutions and unemployment: a critical assessment of the cross-country evidence, in: Howell, D. (Hrsg.): Questioning liberalization: unemployment, labor markets and the welfare state, S. 72-118, Oxford

Bassanini, A./Ernst, E. (2002): Labor market regulation, industrial relations and technological regimes: a tale of comparative advantage, in: Industrial and Corporate Change 11 (3), S. 391-426

Bassanini, A./Nunziata, L./Venn, D. (2009): Job protection and productivity, in: Economic Policy 24 (58), S. 349-402

Belot, M./Boone, J./Van Ours, J. (2002): Welfare effects of employment protection, CEPR Discussion Paper (3396), London

Boeri, T./Garibaldi, P. (2007): Two tier reforms of employment protection: a honeymoon effect?, in: Economic Journal 117 (521), S. 357-385

Booth, A. L./Francesconi, M./Frank, J. (2002): Temporary jobs: Stepping stones or dead ends, in: Economic Journal 112 (480), S. 189-213

Breschi, S./Malerba, L./Orsenigo, L. (2000): Technological regimes and Schumpeterian patterns of innovation, in: Economic Journal 110 (463), S. 288-410 Buchele, R./Christiansen, J. (1999): Labor relations and productivity growth in advanced capitalist economies, in: Review of Radical Political Economics 31 (1), S. $87-110$

Den Hartog, H./Tjan, H. S. (1974): Investeringen, lonen, prijzen en arbeidsplaatsen: CPB Netherlands Bureau for Economic Policy Analysis, Occasional Paper (2), Den Haag

Guichard, S./Rusticelli, E. (2012): Reassessing the NAIRUs after the crisis, OECD Economics Department Working Papers (918)

Hall, P. A./Soskice, D. (2001): Varieties of Capitalism, Oxford

Howell, D. R./Baker, D./Glyn, A./Schmitt, J. (2007): Are protective labor market institutions really at the root of unemployment?, in: Capitalism and Society 2 (1), S. $1-71$

Huselid, M. (1995): The impact of human resource management practices on turnover, productivity and corporate financial performance, in: Academy of Management Journal 38 (3), S. 635-670

Irvin, G. (2011): Inequality and recession in Britain and the US, in: Development and Change 42 (38), S. 154-182

Kleinknecht, A. (1998): Is labour market flexibility harmful to innovation?, Cambridge Journal of Economics 22 (3), S. 387-396 
Kleinknecht, A./Oostendorp, R. M./Pradhan, M. P./Naastepad, C. W. M. (2006): Flexible labour, firm performance and the Dutch job creation miracle, in: Inter national Review of Applied Economics 20 (2), S. 171-187

Lorenz, E. H. (1999): Trust, contract and economic cooperation, in: Cambridge Journal of Economics 23 (3), S. 301-316

Lucidi, F./Kleinknecht, A. (2010): Little innovation, many jobs: An econometric analysis of the Italian labour productivity crisis, in: Cambridge Journal of Economics 34 (3), S. 525-546

Maki, D. M./Palumbo, M. G. (2001): Disentangling the wealth effect: a cohort analysis of household saving in the 1990s: Federal Reserve, Washington, DC

Malcomson, J. M. (1997): Contracts, hold-up, and labor markets, in: Journal of Economic Literature 35 (4), S. 1916-1957

McGinnity, F./Mertens, A. (2004): Wages and wage growth of fixed-term workers in East and West Germany, in: Applied Economics Quarterly 50 (2),

S. $139-163$

Michie, J./Sheehan, M. (2001): Labour market flexibility, human resource management and corporate performance, in: British Journal of Management 12 (4), S. 287-306

Michie, J./Sheehan, M. (2003): Labour market deregulation, flexibility and innovation, in: Cambridge Journal of Economics 27 (1), S.123-143

Muysken, J./van Ardenne, C. H (1976): Den Hartog and Tjan's vintage model as a tool for the determination of structural unemployment, in: De Economist $124(1-2)$, S. $83-102$

Naastepad, C.W. M./Kleinknecht, A. (2004): The Dutch productivity slowdown:The culprit at last?, in: Structural Change and Economic Dynamics 15 (2), S. 137-163 Naastepad, C.W. M./Storm, S. (2006): The innovating firm in a societal context: Labour-management relations and labour productivity, in: Verburg, R. M. Ortt, J. R./Dicke, W. M. (Hrsg.): Managing technology and innovation, London, S. $170-191$

Nickell, S./Layard, R. (1999): Labour market institutions and economic performance, in: Ashenfelter, O./Card, D. (ed.): Handbook of labour economics (ch. 46) Nickell, S./Nunziata, L./Ochel, W. (2005): Unemployment in the OECD since the 1960s. What do we know?, in: Economic Journal 115 (1), S. 1-27

Organisation for Economic Co-operation and Development (OECD) (2003):

Employment Outlook 2003, Paris

Organisation for Economic Co-operation and Development (OECD) (2007):

Employment Outlook 2007, Paris

Palley, T. (2009): America's exhausted paradigm: macroeconomic causes of the financial crisis and the great recession: New America Foundation, New Ameri can Contract Policy Paper, Washington, DC

Picchio, M. (2006): Wage Differentials between Temporary and Permanent Workers in Italy, Quaderni del Dipartimento di Economia dell Universita Politecnica delle Marche (257)

Pieroni, L./Pompei, F. (2008): Evaluating innovation and labour market relationships: the case of Italy, in: Cambridge Journal of Economics 32 (2),

S. $325-47$

Polanyi, M. (1966): The Tacit Dimension, London

Sànchez, R./Toharia, L. (2000): Temporary workers and productivity, in: Applied Economics 32 (5), S. 583-591

Scarpetta, S./Tressel, T. (2004): Boosting productivity via innovation and adoption of new technologies: any role for labor market institutions?: World Bank, Policy Research Working Paper Series (3273), Washington, DC

Schumpeter, J. A. (1912): Theorie der wirtschaftlichen Entwicklung, Leipzig Schumpeter, J. A. (1943): Capitalism, Socialism and Democracy, London Storm, S./Naastepad, C. W. M. (2009): Labor market regulation and productivity growth: evidence for twenty OECD countries (1984-2004), in: Industrial Relations 48 (4), S. 629-654
Storm, S./Naastepad, C. W. M. (2012): Macroeconomics beyond the NAIRU, Cambridge, MA

Sturn, S. (2011): Labour market regimes and unemployment in OECD countries, IMK Working Paper (6), Düsseldorf

Svensson, S. (2011): Flexible working conditions and decreasing levels of trust, in: Employee Relations 34 (2), S. 126-137

Sylos Labini, P. (1984): The Forces of Economic Growth and Decline, Cambridge, MA

Sylos Labini, P. (1993): Progresso Tecnico e Sviluppo Ciclico, Bari Sylos Labini, P. (1999): The employment issue: investment, flexibility and the competition of developing countries, in: BNL Quarterly Review (52) 210, S. $257-280$

Vergeer, R. (2010): Labour market flexibility, productivity and employment, PhD thesis: TU Delft

Vergeer, R./Kleinknecht, A. (2011): The impact of labor market deregulation on productivity: A panel data analysis of 19 OECD countries (1960-2004), in: Journal of Post-Keynesian Economics 33 (2), S. 369-404

Vergeer, R./Kleinknecht, A. (2012a): Do flexible labor markets indeed reduce unemployment?, in: Review of Social Economy, advances access: DOI:10.1080 /00346764.2012.681113 (2. May 2012)

Vergeer, R./Kleinknecht, A. (2012b): Does labor market deregulation reduce labor productivity growth? A panel data analysis of 20 OECD countries (1960-2004), unveröffentlichtes Manuskript:TU Delft, March

Zhou, H./Dekker, R./Kleinknecht, A. (2011): Flexible labor and innovation per formance: evidence from longitudinal firm-level data, in: Industrial and Corporate Change 20 (3), S. $941-968$

\section{AUTOREN}

ALDFRED KLEINKNECHT, Dr., ist Professor für Innovationsökonomie an der TU Delft und zurzeit Senior Fellow im Wirtschafts- und Sozialwissenschaftlichen Institut (WSI) in der Hans-Böckler-Stiftung.

a.h.kleinknecht@tudelft.nl

C. W. M. NAASTEPAD, Dr., ist Dozentin für Volkswirtschaftslehre an derTU Delft.

c.w.m.naastepad@tudelft.nl

SERVAAS STORM, Dr., ist Dozent für Volkswirtschaftslehre an derTU Delft.

s.t.h.storm@tudelft.nl

ROBERT VERGEER, Dr., ist Forscher beiTNO Work \& Employment, eine öffentliche Einrichtung für angewandte Forschung.

robert.vergeer@tno.nl 